\title{
Laplacian on Forms and Anomalies in Closed Hyperbolic Manifolds
}

\author{
A.A. Bytsenko* A. E. Gonçalves, and M. Simões \\ Departamento de Física, Universidade Estadual de Londrina, \\ Caixa Postal 6001, CEP 86051-970, Londrina, PR, Brazil
}

Received 1st June, 1999

\begin{abstract}
The global multiplicative properties of the Laplacian on $j-$ forms and related zeta functions are analyzed. The explicit form of the multiplicative and conformal anomalies in closed oriented hyperbolic manifolds $\Gamma \mathbb{H}^{d}$ are derived.
\end{abstract}

\section{Introduction}

The multiplicative properties of (pseudo-) differential operators as well as properties of their determinants have been studied actively during recent years in the mathematical and physical literature. The anomaly associated with product of regularized determinants of operators can be expressed by means of the noncommutative residue, the Wodzicki residue [1] (see also Refs. $[2,3])$. The Wodzicki residue, which is the unique extension of the Dixmier trace to the wider class of (pseudo-) differential operators $[4,5]$, has been considered within the non-commutative geometrical approach to the standard model of the electroweak interactions $[6,7,8]$ and the Yang-Mills action functional. The product of two (or more) differential operators of Laplace type can arise in higher derivative field theories (for example, in higher derivative quantum gravity).

Some recent papers along these lines can be found in Refs. $[9,10,11,12]$. The zeta function associated to the product of Laplace type operators acting in irreducible rank 1 symmetric spaces and the explicit form of the multiplicative anomaly have been derived in [11].

Under such circumstances we should note that the conformal deformation of a metric and the corresponding conformal anomaly can also play an important role in quantum theories with higher derivatives. It is well known that evaluation of the conformal anomaly is actually possible only for even dimensional spaces and up to now its computation is extremely involved. The general structure of such an anomaly in curved $d$ dimensional spaces (d even) has been studied in [13]. We briefly mention here analysis related to this phe- nomenon for constant curvature spaces. The conformal anomaly calculation for the $d$-dimensional sphere can be found, for example, in Ref. [14]. The explicit computation of the anomaly (of the stress-energy tensor) in irreducible rank 1 symmetric spaces has been carried out in $[15,16,17]$ using the zeta-function regularization and the Selberg trace formula.

The purpose of the present paper is to investigate the spectral zeta functions associated with a product of Laplacians on $j$ - forms and to calculate in an explicit form the multiplicative and conformal anomalies for $d-$ dimensional closed oriented hyperbolic manifolds $\Gamma / \mathbb{H}^{d}$.

\section{The spectral zeta function and the trace formula}

We shall be working with irreducible rank 1 symmetric spaces $X=G / K$ of non-compact type. Thus $G$ will be a connected non-compact simple split rank 1 Lie group with finite center and $K \subset G$ will be a maximal compact subgroup. Up to local isomorphism we choose $X=S O_{1}(d, 1) / S O(d)$. Thus the isotropy group $K$ of the base point $(1,0, \ldots 0)$ is $S O(d) ; X$ can be identified with hyperbolic $d$ - space $\mathbb{H}^{d}, d=\operatorname{dim} X$. It is possible to view $\mathbb{H}^{d}$, for example, as one sheet of the hyperboloid of two sheets in $\mathbb{R}^{d+1}$ given by $q(x)=-x_{0}^{2}+x_{1}^{2}+\ldots+x_{d}^{2}=-1, x_{0}>0$ with the metric induced by the quadratic form $q(x)$. Let $\Gamma \subset G$ be a discrete, co-compact, torsion free subgroup, and let $\chi(\gamma)=\operatorname{trace}(\chi(\gamma))$ be the character of a finitedimensional unitary representation $\chi$ of $\Gamma$ for $\gamma \in \Gamma$. Let $L^{(j)} \equiv \triangle_{\Gamma}^{(j)}$ be the Laplacian on $j$ - forms acting on the vector bundle $V\left(X_{\Gamma}\right)$ over $X_{\Gamma}=\Gamma \backslash G / K$ induced

\footnotetext{
${ }^{*}$ On leave from Sankt-Petersburg State Technical University, Russia
} 
by $\chi$. Note that the non-twisted $j$ - forms on $X_{\Gamma}$ are obtained by taking $\chi=1$. One can define the heat kernel of the elliptic operator $\mathcal{L}^{(j)}=L^{(j)}+b^{(j)}$ by

$$
\operatorname{Tr}\left(e^{-t \mathcal{L}^{(j)}}\right)=\frac{-1}{2 \pi i} \operatorname{Tr} \int_{\mathcal{C}_{0}} e^{-z t}\left(z-\mathcal{L}^{(j)}\right)^{-1} d z
$$

where $\mathcal{C}_{0}$ is an arc in the complex plane $\mathbb{C}$; the $b^{(j)}$ are endomorphisms of the vector bundle $V\left(X_{\Gamma}\right)$. By standard results in operator theory there exist $\varepsilon, \delta>0$ such that for $0<t<\delta$ the heat kernel expansion holds

$$
\omega_{\Gamma}^{(j)}\left(t, b^{(j)}\right)=\sum_{\ell=0}^{\infty} n_{\ell}(\chi) e^{-\left(\lambda_{\ell}^{(j)}+b^{(j)}\right) t}=\sum_{0 \leq \ell \leq \ell_{0}} a_{\ell}\left(\mathcal{L}^{(j)}\right) t^{-\ell}+\mathcal{O}\left(t^{\varepsilon}\right)
$$

where $\left\{\lambda_{\ell}^{(j)}\right\}_{\ell=0}^{\infty}$ is the set of eigenvalues of operator $L^{(j)}$ and $n_{\ell}(\chi)$ denote the multiplicity of $\lambda_{\ell}^{(j)}$. Eventually we would like also to take $b^{(j)}=0$, but for now we consider only non-zero modes: $b^{(j)}+\lambda_{\ell}^{(j)}>0, \forall \ell: \lambda_{0}^{(j)}=0$, $b^{(j)}>0$

Let $a_{0}, n_{0}$ denote the Lie algebras of $A, N$ in an Iwasawa decomposition $G=K A N$. Since the rank of $G$ is $1, \operatorname{dim} a_{0}=1$ by definition, say $a_{0}=\mathbb{R} H_{0}$ for a suitable basis vector $H_{0}$. One can normalize the choice of $H_{0}$ by $\beta\left(H_{0}\right)=1$, where $\beta: a_{0} \rightarrow \mathbb{R}$ is the positive root which defines $n_{0}$; for more detail see Ref. [18]. Since $\Gamma$ is torsion free, each $\gamma \in \Gamma-\{1\}$ can be represented uniquely as some power of a primitive element $\delta: \gamma=\delta^{j(\gamma)}$ where $j(\gamma) \geq 1$ is an integer and $\delta$ cannot be written as $\gamma_{1}^{j}$ for $\gamma_{1} \in \Gamma, j>1$ an integer. Taking $\gamma \in \Gamma, \gamma \neq 1$, one can find $t_{\gamma}>0$ and $m_{\gamma} \in M \stackrel{\text { def }}{=}\left\{m_{\gamma} \in K \mid m_{\gamma} a=a m_{\gamma}, \forall a \in A\right\}$ such that $\gamma$ is $G$ conjugate to $m_{\gamma} \exp \left(t_{\gamma} H_{0}\right)$, namely for some $\mathrm{g} \in G, \mathrm{~g} \gamma \mathrm{g}^{-1}=m_{\gamma} \exp \left(t_{\gamma} H_{0}\right)$. Besides let $\chi_{\sigma}(m)=\operatorname{trace}(\sigma(m))$ be the character of $\sigma$, for $\sigma$ a finite-dimensional representation of $M$.

\section{II.1 Fried's trace formula [19]}

For $0 \leq j \leq d-1$,

$$
\operatorname{Tr}\left(e^{-t \mathcal{L}^{(j)}}\right)=I^{(j)}\left(t, b^{(j)}\right)+I^{(j-1)}\left(t, b^{(j-1)}\right)+H^{(j)}\left(t, b^{(j)}\right)+H^{(j-1)}\left(t, b^{(j-1)}\right)
$$

where

$$
\begin{aligned}
I^{(j)}\left(t, b^{(j)}\right) & \stackrel{\text { def }}{=} \frac{\chi(1) \operatorname{Vol}(\Gamma \backslash G)}{4 \pi} \int_{\mathbb{R}} \mu_{\sigma_{j}}(r) e^{-t\left[r^{2}+b^{(j)}+\left(\rho_{0}-j\right)^{2}\right]} d r, \\
H^{(j)}\left(t, b^{(j)}\right) & \stackrel{\text { def }}{=} \frac{1}{\sqrt{4 \pi t}} \sum_{\gamma \in C_{\Gamma}-\{1\}} \chi(\gamma) t_{\gamma} j(\gamma)^{-1} C(\gamma) \chi_{\sigma_{j}}\left(m_{\gamma}\right) \\
& \times \exp \left\{-\left[b^{(j)} t+\left(\rho_{0}-j\right)^{2} t+\frac{t_{\gamma}^{2}}{4 t}\right]\right\},
\end{aligned}
$$

$\rho_{0}=(d-1) / 2$, and the function $C(\gamma), \gamma \in \Gamma$, defined on $\Gamma-\{1\}$ by

$$
C(\gamma) \stackrel{\text { def }}{=} e^{-\rho_{0} t_{\gamma}}\left|\operatorname{det}_{n_{0}}\left(\operatorname{Ad}\left(m_{\gamma} e^{t_{\gamma} H_{0}}\right)^{-1}-1\right)\right|^{-1}
$$

For Ad denoting the adjoint representation of $G$ on its complexified Lie algebra, one can compute $t_{\gamma}$ as follows [20]

$$
e^{t_{\gamma}}=\max \{|c| \mid c=\text { an eigenvalue of } \operatorname{Ad}(\gamma)\} .
$$

Here $C_{\Gamma}$ is a complete set of representatives in $\Gamma$ of its conjugacy classes; Haar measure on $G$ is suitably normalized. In our case $K \simeq S O(d), M \simeq S O(d-1)$. For $j=0$ (i.e. for smooth functions or smooth vector bun- 
dle sections) the measure $\mu_{0}(r)$ corresponds to the trivial representation of $M$. For $j \geq 1$ there is a measure $\mu_{\sigma}(r)$ corresponding to a general irreducible representation $\sigma$ of $M$. Let $\sigma_{j}$ is the standard representation of $M=S O(d-1)$ on $\Lambda^{j} \mathbb{C}^{(d-1)}$. If $d=2 n$ is even then $\sigma_{j}(0 \leq j \leq d-1)$ is always irreducible; if $d=2 n+1$ the every $\sigma_{j}$ is irreducible except for $j=(d-1) / 2=n$, in which case $\sigma_{n}$ is the direct sum of two $(1 / 2)-$ spin representations $\sigma^{ \pm}: \sigma_{n}=\sigma^{+} \oplus \sigma^{-}$. For $j=n$ the representation $\tau_{n}$ of $K=S O(2 n)$ on $\Lambda^{n} \mathbb{C}^{2 n}$ is not irreducible, $\tau_{n}=\tau_{n}^{+} \oplus \tau_{n}^{-}$is the direct sum of $(1 / 2)-\operatorname{spin}$ representations.

\section{II.2 The Harish-Chandra Plancherel measure}

Let the group $G=S O_{1}(2 n, 1)$. Then

$$
\begin{aligned}
\mu_{\sigma_{j}}(r) & =\left(\begin{array}{c}
2 n-1 \\
j
\end{array}\right) \frac{\pi r}{2^{4 n-4} \Gamma(n)^{2}} \prod_{i=2}^{j+1}\left[r^{2}+\left(n+\frac{3}{2}-i\right)^{2}\right] \\
& \times \prod_{i=j+2}^{n}\left[r^{2}+\left(n+\frac{1}{2}-i\right)^{2}\right] \tanh (\pi r) \text { for } 0 \leq j \leq n-1, \\
\mu_{\sigma_{j}}(r) & =\left(\begin{array}{c}
2 n-1 \\
j
\end{array}\right) \frac{\pi r}{2^{4 n-4} \Gamma(n)^{2}} \prod_{i=2}^{2 n-j}\left[r^{2}+\left(n+\frac{3}{2}-i\right)^{2}\right] \\
\times & \prod_{i=2 n-j+1}^{n}\left[r^{2}+\left(n+\frac{1}{2}-i\right)^{2}\right] \tanh (\pi r) \text { for } n \leq j \leq 2 n-1,
\end{aligned}
$$

and $\mu_{\sigma_{j}}(r)=\mu_{\sigma_{2 n-j-1}}(r)$.

For the group $G=S O_{1}(2 n+1,1)$ one has

$$
\begin{aligned}
& \mu_{\sigma_{j}}(r)=\left(\begin{array}{c}
2 n \\
j
\end{array}\right) \frac{\pi}{2^{4 n-2} \Gamma\left(n+\frac{1}{2}\right)^{2}} \prod_{i=1}^{j+1}\left[r^{2}+(n+1-i)^{2}\right] \\
& \times \prod_{i=j+2}^{n}\left[r^{2}+(n-i)^{2}\right] \text { for } \quad 0 \leq j<n, \\
& \mu_{\sigma_{j}}(r)=\left(\begin{array}{c}
2 n \\
j
\end{array}\right) \frac{\pi}{2^{4 n-2} \Gamma\left(n+\frac{1}{2}\right)^{2}} \prod_{i=1}^{2 n-j+1}\left[r^{2}+(n+1-i)^{2}\right] \\
& \quad \times \quad \prod_{i=2 n-j+2}^{n}\left[r^{2}+(n-i)^{2}\right] \text { for } n+1 \leq j \leq 2 n-1 .
\end{aligned}
$$

We should note that the reason for the pair of terms $\left\{I^{(j)}, I^{(j-1)}\right\}, \quad\left\{H^{(j)}, H^{(j-1)}\right\}$ in the trace formula Eq. (2.3) is that $\tau_{j}$ satisfies $\left.\tau_{j}\right|_{M}=\sigma_{j} \oplus \sigma_{j-1}$.

Finally using Eqs. (2.8)-(2.11) we have

$$
\begin{gathered}
\mu_{\sigma_{j}}(r)=C^{(j)}(d) P(r, d) \times \begin{cases}\tanh (\pi r) & \text { for } d=2 n \\
1 & \text { for } d=2 n+1\end{cases} \\
=C^{(j)}(d) \times\left\{\begin{array}{ll}
\sum_{\ell=0}^{d / 2-1} a_{2 \ell}^{(j)}(d) r^{2 \ell+1} \tanh (\pi r) & \text { for } d=2 n \\
\sum_{\ell=0}^{(d-1) / 2} a_{2 \ell}^{(j)}(d) r^{2 \ell} & \text { for } d=2 n+1
\end{array},\right. \\
C^{(j)}(d)=\left(\begin{array}{c}
d-1 \\
j
\end{array}\right) \frac{\pi}{2^{2 d-4} \Gamma(d / 2)^{2}},
\end{gathered}
$$


where the $P(r, d)$ are even polynomials (with suitable coefficients $\left.a_{2 \ell}^{(j)}(d)\right)$ of degree $d-1$ for $G \neq S O(2 n+1,1)$, and of degree $d=2 n+1$ for $G=S O_{1}(2 n+1,1)[21,18]$.

\section{II.3 Case of the trivial representation}

For $j=0$ we take $I^{(-1)}=H^{(-1)}=0$. Since $\sigma_{0}$ is the trivial representation $\chi_{\sigma_{0}}\left(m_{\gamma}\right)=1$. In this case Fried's formula Eq. (2.3) reduces exactly to the trace formula for $j=0[20,22]$ :

$$
\omega_{\Gamma}^{(0)}\left(t, b^{(0)}\right)=\frac{\chi(1) \operatorname{vol}(\Gamma \backslash G)}{4 \pi} \int_{\mathbb{R}} \mu_{\sigma_{0}}(r) e^{-\left(r^{2}+b^{(0)}+\rho_{0}^{2}\right) t} d r+H^{(0)}\left(t, b^{(0)}\right),
$$

where $\rho_{0}$ is associated with the positive restricted (real) roots of $G$ (with multiplicity) with respect to a nilpotent factor $N$ of $G$ in an Iwasawa decomposition $G=K A N$. The function $H^{(0)}\left(t, b^{(0)}\right)$ has the form

$$
H^{(0)}\left(t, b^{(0)}\right)=\frac{1}{\sqrt{4 \pi t}} \sum_{\gamma \in C_{\Gamma}-\{1\}} \chi(\gamma) t_{\gamma} j(\gamma)^{-1} C(\gamma) e^{-\left[b^{(0)} t+\rho_{0}^{2} t+t_{\gamma}^{2} /(4 t)\right]}
$$

\section{Case of zero modes.}

It can be shown [23] that the Mellin transform of $H^{(0)}(t, 0)\left(b^{(0)}=0\right.$, i.e. the zero modes case)

$$
\mathfrak{H}^{(0)}(s) \stackrel{\text { def }}{=} \int_{0}^{\infty} H^{(0)}(t, 0) t^{s-1} d t
$$

is a holomorphic function on the domain Res $<0$. Then using the result of Refs. [21, 18] one can obtain on Res $<0$,

$$
\begin{aligned}
\mathfrak{H}^{(0)}(s) & =\sum_{\gamma \in C_{\Gamma}-\{1\}} \chi(\gamma) t_{\gamma} j(\gamma)^{-1} C(\gamma) \int_{0}^{\infty} \frac{e^{-\left(\rho_{0}^{2} t+t_{\gamma}^{2} /(4 t)\right)}}{\sqrt{4 \pi t}} t^{s-1} d t \\
& =\frac{\left(2 \rho_{0}\right)^{\frac{1}{2}-s}}{\sqrt{\pi}} \sum_{\gamma \in C_{\Gamma}-\{1\}} \chi(\gamma) t_{\gamma} j(\gamma)^{-1} C(\gamma) t_{\gamma}^{s+\frac{1}{2}} K_{\frac{1}{2}-s}\left(t_{\gamma} \rho_{0}\right),
\end{aligned}
$$

where $K_{\nu}(s)$ is the modified Bessel function, and finally

$$
\mathfrak{H}^{(0)}(s)=\frac{\sin (\pi s)}{\pi} \Gamma(s) \int_{0}^{\infty} \psi_{\Gamma}\left(t+2 \rho_{0} ; \chi\right)\left(2 \rho_{0} t+t^{2}\right)^{-s} d t .
$$

Here $\psi_{\Gamma}(s ; \chi) \equiv d\left(\log Z_{\Gamma}(s ; \chi)\right) / d s$, and $Z_{\Gamma}(s ; \chi)$ is a meromorphic suitably normalized Selberg zeta function [24-29,22,30,21].

\section{The multiplicative anomaly}

In this section the product of the operators on $j$ - forms $\otimes \mathcal{L}_{p}^{(j)}, \mathcal{L}_{p}^{(j)}=L^{(j)}+b_{p}^{(j)}, p=1,2$ will be considered. We are interested in multiplicative properties of determinants, the multiplicative anomaly $[2,3]$. The multiplicative anomaly $F\left(\mathcal{L}_{1}^{(j)}, \mathcal{L}_{2}^{(j)}\right)$ reads

$$
F\left(\mathcal{L}_{1}^{(j)}, \mathcal{L}_{2}^{(j)}\right)=\operatorname{det}_{\zeta}\left[\bigotimes_{p} \mathcal{L}_{p}^{(j)}\right]\left[\operatorname{det}_{\zeta}\left(\mathcal{L}_{1}^{(j)}\right) \operatorname{det}_{\zeta}\left(\mathcal{L}_{2}^{(j)}\right)\right]^{-1}
$$


where we assume a zeta-regularization of determinants, i.e.

$$
\operatorname{det}_{\zeta}\left(\mathcal{L}_{p}^{(j)}\right) \stackrel{\text { def }}{=} \exp \left(-\left.\frac{\partial}{\partial s} \zeta\left(s \mid \mathcal{L}_{p}^{(j)}\right)\right|_{s=0}\right)
$$

Generally speaking, if the anomaly related to elliptic operators is nonvanishing then the relation $\log \operatorname{det}\left(\otimes \mathcal{L}_{p}^{(j)}\right)=\operatorname{Tr} \operatorname{Tog}\left(\otimes \mathcal{L}_{p}^{(j)}\right)$ does not hold.

\section{II.1 The zeta function of the product of Laplacians}

The spectral zeta function associated with the prod- uct $\otimes \mathcal{L}_{p}^{(j)}$ has the form

$$
\zeta\left(s \mid \bigotimes_{p} \mathcal{L}_{p}^{(j)}\right)=\sum_{\ell \geq 0} n_{\ell} \prod_{p}^{2}\left(\lambda_{\ell}^{(j)}+b_{p}^{(j)}\right)^{-s} .
$$

We shall always assume that $b_{1}^{(j)} \neq b_{2}^{(j)}$, say $b_{1}^{(j)}>b_{2}^{(j)}$. If $b_{1}^{(j)}=b_{2}^{(j)}$ then $\zeta\left(s \mid \otimes \mathcal{L}_{p}^{(j)}\right)=\zeta\left(2 s \mid \mathcal{L}^{(j)}\right)$ is a wellknown function. For $b_{1}^{(j)}, b_{2}^{(j)} \in \mathbb{R}$, set $b_{+} \stackrel{\text { def }}{=}\left(b_{1}^{(j)}+\right.$ $\left.b_{2}^{(j)}\right) / 2, b_{-} \stackrel{\text { def }}{=}\left(b_{1}^{(j)}-b_{2}^{(j)}\right) / 2$, thus $b_{1}^{(j)}=b_{+}+b_{-}$and $b_{2}^{(j)}=b_{+}-b_{-}$.

The spectral zeta function can be written as follows [11]:

$$
\zeta\left(s \mid \bigotimes_{p} \mathcal{L}_{p}^{(j)}\right)=\left(2 b_{-}\right)^{\frac{1}{2}-s} \frac{\sqrt{\pi}}{\Gamma(s)} \int_{0}^{\infty} \omega_{\Gamma}^{(j)}\left(t, b_{+}\right) I_{s-\frac{1}{2}}\left(b_{-} t\right) d t
$$

where the integral converges absolutely for $\operatorname{Res}>d / 4$. This formula is a main starting point to study the zeta function. It expresses $\zeta\left(s \mid \otimes \mathcal{L}_{p}^{(j)}\right)$ in terms of the Bessel function $I_{s-\frac{1}{2}}\left(b_{-} t\right)$ and $\omega_{\Gamma}^{(j)}\left(t, b_{+}\right)$, where the trace formula applies to $\omega_{\Gamma}^{(j)}\left(t, b_{+}\right)$. Let $B_{p}(j)=$ $\left(\rho_{0}(p)-j\right)^{2}+b_{p}^{(j)}$ and $A \stackrel{\text { def }}{=} \chi(1) \operatorname{vol}(\Gamma \backslash G) C^{(j)}(d) / 4$.

For Res $>d / 4$ the explicit meromorphic continuation holds:

$$
\begin{gathered}
\zeta\left(s \mid \bigotimes_{p} \mathcal{L}_{p}^{(j)}\right)=A \sum_{\ell=0}^{\frac{d}{2}-1}\left[a_{2 \ell}^{(j)}(d)\left(\mathcal{F}_{\ell}^{(j)}(s)-E_{\ell}^{(j)}(s)\right)\right. \\
\left.+a_{2 \ell}^{(j-1)}(d)\left(\mathcal{F}_{\ell}^{(j-1)}(s)-E_{\ell}^{(j-1)}(s)\right)\right]+\mathcal{I}^{(j)}(s)+\mathcal{I}^{(j-1)}(s),
\end{gathered}
$$

where

$$
E_{\ell}^{(j)}(s) \stackrel{\text { def }}{=} 4 \int_{0}^{\infty} \frac{d r r^{2 j+1}}{1+e^{2 \pi r}} \prod_{p}\left(r^{2}+B_{p}(j)\right)^{-s}
$$

which is an entire function of $s$,

$$
\begin{gathered}
\mathcal{F}_{\ell}^{(j)}(s) \stackrel{\text { def }}{=}\left(B_{1}(j) B_{2}(j)\right)^{-s} \frac{\ell !\left(\frac{2 B_{1}(j) B_{2}(j)}{B_{1}(j)+B_{2}(j)}\right)^{\ell+1}}{(2 s-1)(2 s-2) \ldots(2 s-(\ell+1))} \\
\times F\left(\frac{\ell+1}{2}, \frac{\ell+2}{2} ; s+\frac{1}{2} ;\left(\frac{B_{1}(j)-B_{2}(j)}{B_{1}(j)+B_{2}(j)}\right)^{2}\right), \\
\mathcal{I}^{(j)}(s) \stackrel{\text { def }}{=}\left(2 b_{-}\right)^{\frac{1}{2}-s} \frac{\sqrt{\pi}}{\Gamma(s)} \int_{0}^{\infty} H^{(j)}\left(t, b_{+}\right) I_{s-\frac{1}{2}}\left(b_{-} t\right) t^{s-\frac{1}{2}} d t .
\end{gathered}
$$

and $F(\alpha, \beta ; \gamma ; z)$ is the hypergeometric function.

The goal now is to compute the zeta function and its derivative at $s=0$. Thus we have 


$$
\begin{gathered}
\mathcal{F}_{\ell}^{(j)}(0)=\frac{(-1)^{\ell+1}}{\ell+1}\left(\frac{2 B_{1}(j)}{B_{1}(j)+B_{2}(j)}\right)^{\ell+1} \\
\times F\left(\frac{\ell+1}{2}, \frac{\ell+2}{2} ; \frac{1}{2} ;\left(\frac{B_{1}(j)-B_{2}(j)}{B_{1}(j)+B_{2}(j)}\right)^{2}\right)=\frac{(-1)^{\ell+1}}{2(\ell+1)} \sum_{p}^{2} B_{p}(j)^{\ell+1}, \\
E_{\ell}^{(j)}(0)=4 \int_{0}^{\infty} \frac{d r r^{2 \ell+1}}{1+e^{2 \pi r}}=\frac{(-1)^{\ell}}{\ell+1}\left(1-2^{-2 \ell-1}\right) \mathcal{B}_{2 \ell+2}, \\
\mathcal{I}^{(j)}(0)=0,
\end{gathered}
$$

where $\mathcal{B}_{2 n}$ are the Bernoulli numbers.

A preliminary form of the zeta function $\zeta\left(s \mid \bigotimes_{p} \mathcal{L}_{p}^{(j)}\right)$ at $s=0$ is

$$
\begin{gathered}
\zeta\left(0 \mid \bigotimes_{p} \mathcal{L}_{p}^{(j)}\right)=A \sum_{\ell=0}^{\frac{d}{2}-1} \frac{(-1)^{\ell+1}}{2(\ell+1)}\left[\sum _ { p } \left(a_{2 \ell}^{(j)}(d) B_{p}(j)^{\ell+1}\right.\right. \\
\left.\left.+a_{2 \ell}^{(j-1)}(d) B_{p}(j-1)^{\ell+1}\right)+\left(2-2^{-2 \ell}\right) \mathcal{B}_{2 \ell+2}\left(a_{2 \ell}^{(j)}(d)+a_{2 \ell}^{(j-1)}(d)\right)\right] .
\end{gathered}
$$

The derivative of the zeta function at $s=0$ has the form:

$$
\zeta^{\prime}\left(0 \mid \bigotimes_{p} \mathcal{L}_{p}^{(j)}\right)=A \sum_{\ell=0}^{\frac{d}{2}-1}\left[\sum_{m}^{4}\left(a_{2 \ell}^{(j)}(d) \mathcal{E}_{m}^{(j)}+a_{2 \ell}^{(j-1)}(d) \mathcal{E}_{m}^{(j-1)}\right)\right]
$$

where

$$
\begin{aligned}
& \mathcal{E}_{1}^{(j)}= \ell !\left(B_{1}(j)^{\ell+1}+B_{2}(j)^{\ell+1}\right) \sum_{k=0}^{\ell} \frac{(-1)^{k+1}}{k !(\ell-k) !(j+1-k) !}, \\
& \mathcal{E}_{2}^{(j)}= B_{2}(j)^{\ell+1}\left(\frac{B_{1}(j)-B_{2}(j)}{2 B_{1}(j)}\right) \frac{(-1)^{\ell}}{(\ell+1) !} \sum_{k=1}^{\infty} \frac{(\ell+k+1) !}{(k+1) !} \\
& \times \sigma_{n}\left(\frac{B_{1}(j)-B_{2}(j)}{B_{1}(j)}\right)^{k}, \\
& \mathcal{E}_{3}^{(j)}= \log \left(B_{1}(j) B_{2}(j)\right) \frac{(-1)^{\ell}}{2(\ell+1)}\left(B_{1}(j)^{\ell+1}+B_{2}(j)^{\ell+1}\right) \\
&-4 \int_{0}^{\infty} \frac{r^{2 \ell+1} \log \left(\frac{r^{2}+B_{1}(j)}{r^{2}+B_{2}(j)}\right) d r}{1+e^{2 \pi r}}, \\
&\left.\mathcal{E}_{4}^{(j)} \equiv \frac{d}{d s} \mathcal{I}^{(j)}(s)\right|_{s=0}=\int_{0}^{\infty}\left[H^{(j)}\left(t, b_{1}^{(j)}\right)+H^{(j)}\left(t, b_{2}^{(j)}\right)\right] t^{-1} d t,
\end{aligned}
$$

and $\sigma_{n} \stackrel{\text { def }}{=} \sum_{k=1}^{n} k^{-1}$.

\section{III.2 The one-loop effective action}

After a standard integration, the contribution to the Euclidean one-loop effective action can be written as follows:

$$
\mathfrak{W}^{(1)}=\frac{1}{2} \log \operatorname{det}\left(\bigotimes_{p} \mathcal{L}_{p}^{(j)} \mu^{-2}\right)=-\frac{1}{2}\left[\zeta^{\prime}\left(0 \mid \bigotimes_{p} \mathcal{L}_{p}^{(j)}\right)+\log \mu^{2} \zeta\left(0 \mid \bigotimes_{p} \mathcal{L}_{p}^{(j)}\right)\right]
$$

where $\mu^{2}$ is a normalization parameter. As a result we have 


$$
\begin{gathered}
\mathfrak{W}^{(1)}=-\frac{1}{2} A \sum_{\ell=0}^{\frac{d}{2}-1}\left[a_{2 \ell}^{(j)}(d)\left(\sum_{m}^{4} \mathcal{E}_{m}^{(j)}+\log \mu^{2}\left(\mathcal{F}_{\ell}^{(j)}(0)-E_{\ell}^{(j)}(0)\right)\right)\right. \\
\left.+a_{2 \ell}^{(j-1)}(d)\left(\sum_{m}^{4} \mathcal{E}_{m}^{(j-1)}+\log \mu^{2}\left(\mathcal{F}_{\ell}^{(j-1)}(0)-E_{\ell}^{(j-1)}(0)\right)\right)\right]
\end{gathered}
$$

where $\mathcal{F}_{\ell}^{(j)}(0), E_{\ell}^{(j)}(0)$ and $\mathcal{E}_{m}^{(j)}$ are given by the formulae (3.9), (3.10) and (3.14)-(3.17) respectively.

\section{III.3 The residue formula and the multi- plicative anomaly}

The value of $F\left(\mathcal{L}_{1}, \mathcal{L}_{2}\right)$ can be expressed by means of the non-commutative Wodzicki residue [1]. Let $\mathcal{O}_{p}, p=1,2$, be invertible elliptic (pseudo-) differential operators of real non-zero orders $\alpha$ and $\beta$ such that $\alpha+\beta \neq 0$. Even if the zeta functions for operators $\mathcal{O}_{1}, \mathcal{O}_{2}$ and $\mathcal{O}_{1} \otimes \mathcal{O}_{2}$ are well defined and if their principal symbols satisfy the Agmon-Nirenberg condition (with appropriate spectra cuts) one has in general that $F\left(\mathcal{O}_{1}, \mathcal{O}_{2}\right) \neq 1$. For such invertible elliptic operators the formula for the anomaly of commuting operators holds:

$$
\mathcal{A}\left(\mathcal{O}_{1}, \mathcal{O}_{2}\right)=\mathcal{A}\left(\mathcal{O}_{2}, \mathcal{O}_{1}\right)=\log \left(F\left(\mathcal{O}_{1}, \mathcal{O}_{2}\right)\right)=\frac{\operatorname{res}\left[\left(\log \left(\mathcal{O}_{1}^{\beta} \otimes \mathcal{O}_{2}^{-\alpha}\right)\right)^{2}\right]}{2 \alpha \beta(\alpha+\beta)} .
$$

More general formulae have been derived in Refs. [2,3]. Furthermore the anomaly can be iterated consistently. Indeed, using Eq. (3.20) we have

$$
\begin{aligned}
\mathcal{A}\left(\mathcal{O}_{1}, \mathcal{O}_{2}\right) & =\zeta^{\prime}\left(0 \mid \mathcal{O}_{1} \mathcal{O}_{2}\right)-\zeta^{\prime}\left(0 \mid \mathcal{O}_{1}\right)-\zeta^{\prime}\left(0 \mid \mathcal{O}_{2}\right) \\
\mathcal{A}\left(\mathcal{O}_{1}, \mathcal{O}_{2}, \mathcal{O}_{3}\right)= & \zeta^{\prime}\left(0 \mid \bigotimes_{j}^{3} \mathcal{O}_{j}\right)-\sum_{j}^{3} \zeta^{\prime}\left(0 \mid \mathcal{O}_{j}\right)-\mathcal{A}\left(\mathcal{O}_{1}, \mathcal{O}_{2}\right), \\
\cdot & \cdot \cdot \cdot \cdot \\
\mathcal{A}\left(\mathcal{O}_{1}, \mathcal{O}_{2}, \ldots, \mathcal{O}_{n}\right)= & \zeta^{\prime}\left(0 \mid \bigotimes_{j}^{n} \mathcal{O}_{j}\right)-\sum_{j}^{n} \zeta^{\prime}\left(0 \mid \mathcal{O}_{j}\right)-\mathcal{A}\left(\mathcal{O}_{1}, \mathcal{O}_{2}\right) \\
& -\mathcal{A}\left(\mathcal{O}_{1}, \mathcal{O}_{2}, \mathcal{O}_{3}\right) \ldots-\mathcal{A}\left(\mathcal{O}_{1}, \mathcal{O}_{2}, \ldots, \mathcal{O}_{n-1}\right) .
\end{aligned}
$$

\section{III.4 The explicit formula for the multiplicative anomaly}

In particular, for $n=2$ and $\mathcal{O}_{p} \equiv \mathcal{L}_{p}^{(j)}$ the anomaly is given by the following formula

$$
\mathcal{A}\left(\mathcal{L}_{1}^{(j)}, \mathcal{L}_{2}^{(j)}\right)=A \sum_{\ell=0}^{\frac{d}{2}-1}\left[\Omega_{\ell}^{(j)}+\Omega_{\ell}^{(j-1)}\right]
$$

where

$$
\begin{gathered}
\Omega_{\ell}^{(j)}=\frac{a_{2 \ell}^{(j)}(d)(-1)^{\ell}}{2}\left[\frac{\ell}{2}\left(B_{1}(j)-B_{2}(j)\right)^{2} B_{2}(j)^{\ell-1}\right. \\
+\frac{\ell(\ell-1)}{4}\left(B_{1}(j)-B_{2}(j)\right)^{3} B_{2}(j)^{\ell-2}+\sum_{p=3}^{\ell} \frac{\ell !}{(p+1) p !(\ell-p) !} \\
\left.\times\left(\frac{1}{p}+\frac{1}{p-1}+\sum_{q=1}^{p-2} \frac{1}{p-q-1}\right)\left(B_{1}(j)-B_{2}(j)\right)^{p+1} B_{2}(j)^{\ell-p}\right] .
\end{gathered}
$$

We note that for the four-dimensional space with $G=S O_{1}(4,1)$, one derives from Eq. (3.22) the result 


$$
\mathcal{A}\left(\mathcal{L}_{1}^{(j)}, \mathcal{L}_{2}^{(j)}\right)=-A_{G}^{(j)}\left(b_{1}^{(j)}-b_{1}^{(j)}\right)^{2}-A_{G}^{(j-1)}\left(b_{1}^{(j-1)}-b_{1}^{(j-1)}\right)^{2}
$$

which also follows from Wodzicki's formula (3.20), where we should set $A_{G}^{(j)}=A a_{21}^{(j)}(4) / 4$.

\section{The conformal anomaly and associated operator prod- ucts.}

In this section we start with a conformal deformation of a metric and the conformal anomaly of the energy stress tensor. It is well known that (pseudo-) Rieman- nian metrics $\mathrm{g}_{\mu \nu}(x)$ and $\tilde{\mathrm{g}}_{\mu \nu}(x)$ on a manifold $X$ are (pointwise) conformal if $\tilde{\mathrm{g}}_{\mu \nu}(x)=\exp (2 f) \mathrm{g}_{\mu \nu}(x), f \in$ $C^{\infty}(\mathbb{R})$. For constant conformal deformations the variation of the connected vacuum functional (the effective action) can be expressed in terms of the generalized zeta function related to an elliptic self-adjoint operator $\mathcal{O}[31]:$

$$
\delta W=-\zeta(0 \mid \mathcal{O}) \log \mu^{2}=\int_{X_{\Gamma}}<T_{\mu \nu}(x)>\delta \mathrm{g}^{\mu \nu}(x) d x
$$

where $\left\langle T_{\mu \nu}(x)>\right.$ means that all connected vacuum graphs of the stress-energy tensor $T_{\mu \nu}(x)$ are to be included. Therefore Eq. (4.1) leads to

$$
<T_{\mu}^{\mu}(x)>=\left(\operatorname{Vol}\left(X_{\Gamma}\right)\right)^{-1} \zeta(0 \mid \mathcal{O}) .
$$

The formulae (3.5), (3.9), (3.10) and (3.11) give an explicit result for the conformal anomaly, namely

$$
\begin{gathered}
<T_{\mu}^{\mu}(x)>_{\left(\mathcal{O}=\bigotimes \mathcal{L}_{p}^{(j)}\right)}=\frac{1}{(4 \pi)^{d / 2} \Gamma(d / 2)} \sum_{\ell=0}^{\frac{d}{2}-1} \frac{(-1)^{\ell+1}}{2(\ell+1)}\left\{\sum _ { p } \left[a_{2 \ell}^{(j)}(d) B_{p}(j)^{\ell+1}\right.\right. \\
\left.\left.+a_{2 \ell}^{(j-1)}(d) B_{p}(j-1)^{\ell+1}\right]+\left(2-2^{-2 \ell}\right) \mathcal{B}_{2 \ell+2}\left(a_{2 \ell}^{(j)}(d)+a_{2 \ell}^{(j-1)}(d)\right)\right\},
\end{gathered}
$$

where $d$ is even. For $B_{1,2}(j)=B(j), B_{1,2}(j-1)=B(j-1)$ the anomaly (4.3) has the form

$$
\begin{aligned}
< & \left.T_{\mu}^{\mu}(x)>_{\left(\mathcal{L}^{(j)}\right.} \otimes \mathcal{L}^{(j)}\right) \\
& =\frac{1}{(4 \pi)^{d / 2} \Gamma(d / 2)} \sum_{\ell=0}^{\frac{d}{2}-1} \frac{(-1)^{\ell+1}}{2(\ell+1)}\left\{\left[a_{2 \ell}^{(j)}(d) B(j)^{\ell+1}\right.\right. \\
& \left.\left.+a_{2 \ell}^{(j-1)}(d) B(j-1)^{\ell+1}\right]+\left(2-2^{-2 \ell}\right) \mathcal{B}_{2 \ell+2}\left(a_{2 \ell}^{(j)}(d)+a_{2 \ell}^{(j-1)}(d)\right)\right\} .
\end{aligned}
$$

Note that for a minimally coupled scalar field of mass $m, B(0)=\rho_{0}^{2}+m^{2}$. The simplest case is, for example, $G=$ $S O_{1}(2,1) \simeq S L(2, \mathbb{R})$; besides $X=\mathbb{H}^{2}$ is a two-dimensional real hyperbolic space. Then we have $\rho_{0}^{2}=1 / 4, a_{20}^{(0)}=1$, and finally

$$
\left.<T_{\mu}^{\nu}\left(x \in \Gamma \backslash \mathbb{H}^{2}\right)>_{\left(\mathcal{L}^{(0)}\right.} \otimes \mathcal{L}^{(0)}\right)=-\frac{1}{4 \pi}\left(b+\frac{1}{3}\right)
$$

For real $d$-dimensional hyperbolic space the scalar curvature is $R(x)=-d(d-1)$. In the case of the conformally invariant scalar field we have $B(0)=\rho_{o}^{2}+R(x)(d-2) /[4(d-1)]$. As a consequence, $B(0)=1 / 4$ and

$$
\begin{aligned}
<T_{\mu}^{\mu}\left(x \in \Gamma \backslash \mathbb{H}^{d}\right) & \left.>{ }_{\left(\mathcal{L}^{(0)}\right.} \otimes \mathcal{L}^{(0)}\right) \\
& \times \frac{1}{(4 \pi)^{d / 2} \Gamma(d / 2)} \sum_{\ell=0}^{\frac{d}{2}-1} \frac{(-1)^{\ell+1}}{\ell+1} a_{2 \ell}^{(0)}(d) \\
& \times\left\{2^{-2 \ell-2}+\left(1-2^{-2 \ell-1}\right) \mathcal{B}_{2 \ell+2}\right\} .
\end{aligned}
$$


Thus in conformally invariant scalar theory the anomaly of the stress tensor coincides with one associated with operator product. This statement holds not only for hyperbolic spaces considered above but for all constant curvature manifolds as well [16].

\section{Concluding remarks}

In this paper the one-loop contribution to the effective action (3.19), the multiplicative anomaly (3.22) and the conformal anomaly of the stress-energy momentum tensor (4.4), related to the operator product, have been evaluated explicitly. In addition we have considered the product $\bigotimes_{p} \mathcal{L}_{p}^{(j)}$ of Laplace operators $\mathcal{L}_{p}^{(j)}$ acting in closed real hyperbolic manifolds. Note that the multiplicative anomaly is equal to zero for $d=2$ and for the odd dimensional cases. It seems to us that the explicit results for the anomalies are not only interesting as mathematical results but are of physical interest. We hope that proposed discussion will be interesting in view of future applications to concrete problems in quantum field theory.

\section{Acknowledgments}

We thank F. L. Williams for useful discussion. First author partially supported by a CNPq grant (Brazil), RFFI grant (Russia) No 98-02-18380-a, and by GRACENAS grant (Russia) No 6-18-1997.

\section{References}

[1] M. Wodzicki, "Non-Commutative Residue. Chapter I". In Lecture Notes in Mathematics, Yu.I. Manin, Editor, Springer-Verlag, Berlin, 1289, 320 (1987).

[2] M. Kontsevich and S. Vishik, "Determinants of Elliptic Pseudo-Differential Operators", Preprint MPI/9430 (1994).

[3] M. Kontsevich and S. Vishik, "Geometry of Determinants of Elliptic Operators", hep-th/9406140 (1994).

[4] A. Connes, Commun. Math. Phys. 117, 673 (1988).

[5] D. Kastler, Commun. Math. Phys. 166, 633 (1995).

[6] A. Connes and J. Lott, Nucl. Phys. B 18, 29 (1990).

[7] A. Connes, "Non-Commutative Geometry", Academic Press, New York (1994).
[8] A. Connes, Commun. Math. Phys. 182, 155 (1996).

[9] W. Kalau and M. Walze, J. Geom. Phys. 16, 327 (1995).

[10] E. Elizalde, L. Vanzo and S. Zerbini, Commun. Math. Phys. 194, 613 (1998).

[11] A.A. Bytsenko and F.L. Williams, JMP 39, 1075 (1998).

[12] E. Elizalde, G. Cognola and S. Zerbini, Nucl. Phys. B 532, 407 (1998).

[13] S. Deser and A. Schwimmer, Phys. Lett. B 309, 279 (1993).

[14] E. Copeland and D. Toms, Class. Quant. Grav. 3, 431 (1986).

[15] A. A. Bytsenko, E. Elizalde and S.D. Odintsov, JMP 36, 5084 (1995)

[16] A. A. Bytsenko, A.E. Gonçalves and F.L. Williams, Mod. Phys. Lett. A 13, 99 (1998).

[17] A. A. Bytsenko, A.E. Gonçalves and F.L. Williams, JETP Lett. 67, 176 (1998).

[18] F. L. Williams, JMP 38, 796 (1997).

[19] D. Fried, Invent. Math. 84, 523 (1986).

[20] N. Wallach, Bull. Am. Math. Soc. 82, 171 (1976).

[21] A. A. Bytsenko, G. Cognola, L. Vanzo and S. Zerbini, Phys. Rep. 266, 1 (1996).

[22] F. Williams, "Lectures on the Spectrum of $L^{2}(\Gamma \backslash G)$ ", Pitman Rearch Notes in Math. 242, Longman House Pub. (1990).

[23] F. Williams, Pacific J. of Math. 182, 137 (1998).

[24] A. Selberg, J. India Math. Soc. 20, 47 (1956).

[25] D. Fried, Ann.Sci. E'cole Norm. Sup. 10, 133 (1977).

[26] R. Gangolli, Illinois J. Math. 21, 1 (1977).

[27] R. Gangolli and G. Warner, Nagoya Math. J. 78, 1 (1980).

[28] D. Scott, Math. Ann. 253, 177 (1980).

[29] M. Wakayama, Hiroshima Math. J. 15, 235 (1985).

[30] F. Williams, "Some Zeta Functions Attached to $\Gamma \backslash G / K$ ", in New Developments in Lie Theory and Their Applications, Edited by J. Tirao and N. Wallach, Birkhäuser Progress in Math. Ser. 105, 163 (1992).

[31] N. D. Birrell and P.C.W. Davies, "Quantum Fields in Curved Space", Cambridge University Press, Cambridge (1982). 